\title{
EFICIÊNCIA DE ABSORÇÃO, TRANSPORTE E UTILIZAČ̃̃O DE MACRONUTRIENTES POR PORTA-ENXERTOS DE CARAMBOLEIRA, CULTIVADOS EM SOLUÇÕES NUTRITIVAS ${ }^{1}$
}

\author{
Up take, transportat and use of macronutrients in rootstock for star \\ fruit tree cultivated in nutrient solutions
}

\author{
Danilo Eduardo Rozane ${ }^{2}$, Renato de Mello Prado , Claudenir Facincani Franco ${ }^{4}$, William Natale ${ }^{5}$
}

\begin{abstract}
RESUMO
O estado nutricional de plantas cultivadas em solução nutritiva pode ser influenciado pelo tipo de solução utilizada, afetando desta forma seu adequado crescimento. Com este trabalho, objetivou-se avaliar a exigência nutricional de porta-enxertos de caramboleira, cultivados em quatro diferentes soluções nutritivas. O delineamento experimental empregado foi inteiramente casualizado, com três repetições, tendo como tratamentos as quatro soluções. Como solução padrão foi utilizada a de Hoagland \& Arnon, comparada à outras três soluções. O experimento foi conduzido em condições de casa-de-vegetação, em recipientes plásticos com $8 \mathrm{~L}$ de capacidade. Após 150 dias do transplantio, foram determinados a matéria seca e o teor de nutrientes, inferindo-se o conteúdo dos macronutrientes na planta. Com esses resultados calculou-se os índices: eficiência de absorção; eficiência de transporte e eficiência de utilização dos nutrientes. As soluções nutritivas de Sarruge, de Castellane \& Araújo e de Furlani foram semelhantes na produção de matéria seca das plantas de caramboleira. O uso da solução nutritiva de Hoagland \& Arnon resultou em menor produção de matéria seca das caramboleiras, e, também, menor eficiência de utilização de Ca e Mg pelas plantas. A eficiência de transporte dos nutrientes foi a mais afetada pelas soluções nutritivas utilizadas, enquanto a eficiência de utilização dos nutrientes sofreu pouca variação em função das soluções empregadas.
\end{abstract}

Termos para indexação: Averrhoa carambola, frutíferas, mobilidade, nutrição mineral, produção de mudas.

\begin{abstract}
The nutritional status of plants cultivated in nutrient solutions way be influenced by the solution which would affect its growth plants. The present essay was undergone to assess the nutritional demands of root stock for star fruit trees, cultivated in four different nutritional solutions. An completely experimental design was used with three repetitions, with four different nutrient solutions as treatments. The Hoagland \& Arnon solution was used as standard and was the control wich was with compared three other solutions. The experiment was carrie out under green house conditions, using $8 \mathrm{~L}$ pot. After 150 days of transplantation, dry material and macro nutrient levels were determined. The following indicators were calculated: absorption efficiency; transportation efficiency, and efficiency of nutrient use. The Sarruge, the Castellane \& Araújo, and the Furlani nutrient solutions were similar to accumulation of dry material in the star fruit plants. The use of the Hoagland \& Arnon solution resulted in lower material dry production in and also lower of $\mathrm{Ca}$ and $\mathrm{Mg}$ use. Nutrient transportation efficiency was the indicator most affected by the different nutritional solutions used, while efficiency of nutrient use showed small variation when comparing the solutions.
\end{abstract}

Index terms: Averrhoa carambola, fruit plants, mobility, mineral nutrition, seedling production.

(Recebido em 31 de julho de 2006 e aprovado em 6 de fevereiro de 2007)

\section{INTRODUÇÃO}

A produção das culturas é afetada pela eficiência nutricional. $\mathrm{O}$ termo eficiência nutricional está relacionado às eficiências: de absorção que indica a capacidade da planta em "extrair" nutrientes do meio de cultivo (solução nutritiva). Salienta-se que os mecanismos desenvolvidos pelas plantas para alta eficiência de absorção diferem entre as espécies. Algumas produzem extenso sistema radicular, enquanto outras têm alta taxa de absorção por unidade de comprimento de raiz, ou seja, alto influxo de nutrientes (Föhse et al. 1988); de transporte que indica a capacidade da planta em converter o nutriente absorvido em matéria seca; de uso ou utilização que segundo Gerloff \& Gabelman (1983) é a capacidade de uma planta redistribuir e reutilizar os elementos minerais de um órgão mais velho e senescente caracteriza a eficiência de uso no metabolismo do processo de crescimento. Este processo pode ser chamado de eficiência de utilização. Lauchli (1987) complementa que a

\footnotetext{
${ }^{1}$ Projeto com Auxílio à Pesquisa FAPESP Proc. 04/09560-0

${ }^{2}$ Engenheiro Agrônomo, Doutorando - Departamento de Solos e Adubos - Universidade Estadual Paulista/UNESP - Via de Acesso Professor Paulo Donato Castellane, s/n - 14870-000 - Jaboticabal, SP - danilorozane@yahoo.com.br - Bolsista da FAPESP Proc. 06/55570-2

${ }^{3}$ Engenheiro Agrônomo, Doutor, Professor - Departamento de Solos e Adubos - Universidade Estadual Paulista/UNESP - Campus de Jaboticabal - Via de Acesso Professor Paulo Donato Castellane, s/n - 14870-000 - Jaboticabal, SP - rmprado@fcav.unesp.br - Bolsista do CNPq ${ }^{4}$ Engenheiro Agrônomo, Mestre - Departamento de Solos e Adubos - Universidade Estadual Paulista/UNESP - Campus de Jaboticabal - Via de Acesso Professor Paulo Donato Castellane, s/n - 14870-000 - Jaboticabal, SP - cfafranco@hotmail.com

${ }^{5}$ Engenheiro Agrônomo, Doutor, Professor Adjunto - Departamento de Solos e Adubos - Universidade Estadual Paulista/UNESP - Campus de Jaboticabal - Via de Acesso Professor Paulo Donato Castellane, s/n - 14870-000 - Jaboticabal, SP - natale@fcav.unesp.br - Bolsista do CNPq.
} 
eficiência de uso de um nutriente é definida como a relação entre a concentração do nutriente no tecido e a produção, enquanto para Graham (1984), eficiência é a habilidade de um genótipo em fornecer altas produções num ambiente deficiente no nutriente em estudo.

Segundo Marschner (1995), as diferenças genotípicas quanto à eficiência nutricional ocorrem por várias razões, as quais estão relacionadas à absorção, ao transporte e à utilização dos nutrientes pelas plantas. Essas diferenças genotípicas envolvidas na nutrição mineral podem ser explicadas, de acordo com Gerloff \& Gabelman (1983), por aspectos morfológicos e fisiológicos relacionados à absorção de nutrientes. Dentre os aspectos morfológicos tem-se: diâmetro e comprimento de raízes, formação de pêlos radiculares e relação superfície de raiz/ unidade de peso da parte aérea. Os aspectos fisiológicos incluem os fatores que descrevem a cinética de absorção, incluindo Imax (taxa máxima de influxo líquido), Km (constante de Michaelis-Menten) e Cmin (concentração mínima abaixo da qual não mais ocorre influxo líquido).

O cultivo de plantas em vasos, utilizando-se de solução nutritiva, é uma ferramenta muito útil nos estudos de nutrição mineral e na busca de soluções e genótipos mais eficientes. Com a melhoria da eficiência nutricional, aumenta-se a produtividade e reduz-se o emprego de adubos, com conseqüiente diminuição nos custos. Assim, a otimização da eficiência nutricional é de grande importância, pois os fertilizantes contribuem com aproximadamente $30 \%$ do custo total da produção (Fageria, 1998).

Como resultado da constante interação entre as fases sólida e líquida do solo, juntamente à intensa atividade de raízes e microorganismos, à medida que os mesmos retiram água, nutrientes e oxigênio, ocorre liberação de dióxido de carbono e uma grande quantidade de compostos orgânicos, para solução do solo que variam de uma região à outra do sistema radicular e a cada momento (Epstein \& Bloom, 2006).

Assim, a escolha de meios artificiais mais simples permite melhor controle das proporções dos diversos nutrientes, visto que a concentração de elementos na solução nutritiva é proporcional àquela encontrada próxima das raízes das plantas (Adams, 1994).

Lucena (1997) complementa que resultados obtidos em sistemas hidropônicos são mais precisos que aqueles obtidos em cultivo no solo, além de representar menor custo em termos de análises químicas.

$\mathrm{Na}$ literatura existem várias indicações de soluções nutritivas para o cultivo de plantas, entretanto, inexistem estudos que apontem a solução nutritiva ideal para o cultivo de mudas de caramboleira. Sabe-se que as eficiências nutricionais variam em função das modificações genéticas de cada planta, sendo uma interessante ferramenta para explicar a produção de matéria seca e, conseqüentemente, o aumento de produção. Diante disto, com a presente pesquisa objetivou-se estudar a produção de matéria seca das plantas e as eficiências de absorção, de transporte e de utilização de porta-enxertos de caramboleira, em diferentes soluções nutritivas.

\section{MATERIAL E MÉTODOS}

O experimento foi realizado em casa-de-vegetação, sob hidroponia, na FCAV/Unesp campus Jaboticabal-SP.

Para a produção dos porta-enxertos de caramboleira (Averrhoa carambola L.) foi utilizada a propagação por sementes, como indicado por Donadio et al. (2001). Para isto, foram escolhidos frutos em um pomar de caramboleira adulta (cultivar Malásia), dos quais extraiu-se as sementes, que após germinarem, permaneceram por mais 4 meses em tubetes preenchidos com substrato à base de casca de pinus e vermiculita, para posterior transplantio para as soluções hidropônicas.

O delineamento experimental empregado foi inteiramente casualizado, com três repetições, sendo cada repetição a média de quatro plantas, tendo-se como tratamentos as quatro soluções nutritivas. Como solução padrão foi utilizada a de Hoagland \& Arnon (1950), comparada a outras três soluções: Sarruge (1975), Castellane \& Araújo (1995) e Furlani et al. (1999), apresentam as concentrações de nutrientes indicadas na Tabela 1.

Durante os primeiros 15 dias após o transplante, os porta-enxertos foram mantidos nas respectivas soluções nutritivas, diluídas a $1 \frac{1}{4}$ da concentração preconizada pelos autores. Após esse período de adaptação, as plantas foram submetidas às soluções originais, até 150 dias após o transplante, acondicionadas em recipientes plásticos (polipropileno virgem), com $8 \mathrm{~L}$ de capacidade. Sendo tais soluções originais renovadas a cada 25 dias.

Ressalta-se que, para o manejo das soluções nutritivas ao longo do período de estudo, o $\mathrm{pH}$ foi monitorado diariamente, ajustando-se a 5,5 $\pm 0,5$, usandose solução $\mathrm{NaOH}$ ou $\mathrm{HCl} 0,1$ M.L ${ }^{-1}$. A condutividade elétrica das soluções nutritivas esteve na faixa de 1,8 a $1,9 \mathrm{dS} . \mathrm{m}^{-1} \mathrm{e}$ não variou dentre o período de troca ( 25 dias), indicando que a troca da solução nutritiva a cada 25 dias foi suficiente para manter a condutividade elétrica próxima de $2,4 \mathrm{dS} \mathrm{m}{ }^{-1}$, conforme indicação de Távora et al. (2001) para o cultivo de mudas de goiabeira, uma vez que inexiste indicações específicas para a caramboleira. 
TABELA 1 - Composição química dos nutrientes presentes na solução nutritiva indicada por Hoagland \& Arnon (1950); Sarruge (1975); Castellane \& Araújo (1995) e Furlani et al. (1999).

\begin{tabular}{|c|c|c|c|c|}
\hline & Hoagland \& Arnon (1950) & Sarruge (1975) & Castellane \& Araújo (1995) & Furlani (1999) \\
\hline \multicolumn{5}{|c|}{ 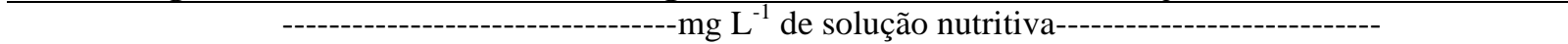 } \\
\hline $\mathrm{N}$ & 210,1 & 210,1 & 222,5 & 202,0 \\
\hline $\mathrm{P}$ & 31,0 & 31,0 & 61,9 & 31,5 \\
\hline K & 234,6 & 234,6 & 426,2 & 193,4 \\
\hline $\mathrm{Ca}$ & 200,4 & 200,4 & 139,9 & 142,5 \\
\hline $\mathrm{Mg}$ & 48,6 & 48,6 & 24,3 & 39,4 \\
\hline $\mathrm{S}$ & 64,2 & 64,1 & 32,4 & 52,3 \\
\hline \multicolumn{5}{|c|}{ 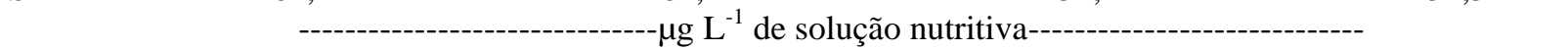 } \\
\hline B & 500 & 500 & 498 & 262 \\
\hline $\mathrm{Cu}$ & 20 & 39 & 48 & 38 \\
\hline $\mathrm{Cl}$ & 648 & 722 & - & - \\
\hline $\mathrm{Fe}$ & 5022 & 5000 & 5000 & 1800 \\
\hline Mn & 502 & 502 & 419 & 369 \\
\hline Mo & 11 & 12 & 52 & 65 \\
\hline $\mathrm{Zn}$ & 50 & 98 & 261 & 114 \\
\hline
\end{tabular}

Para a reposição da água evapotranspirada foi utilizada água destilada, sendo a solução nutritiva oxigenada constantemente e renovada quinzenalmente.

Na colheita, as plantas foram divididas em raízes, caule e folhas. Depois, todo o material vegetal foi lavado em água destilada, seco em estufa com circulação de ar à temperatura de 65 a $70^{\circ} \mathrm{C}$, até peso constante.

A partir da matéria seca e do conteúdo dos nutrientes na planta, determinados segundo metodologia descrita por Bataglia et al. (1983), foram calculados os índices: (a) eficiência de absorção = (conteúdo total do nutriente na planta)/(matéria seca de raízes), conforme Swiader et al. (1994); (b) eficiência de transporte = ((conteúdo do nutriente na parte aérea)/(conteúdo total do nutriente na planta)) x 100, de acordo com Li et al. (1991); (c) eficiência de utilização $=(\text { matéria seca total produzida })^{2} /$ (conteúdo total do nutriente na planta), segundo Siddiqi \& Glass (1981).

Realizaram-se análises de variância e comparação de médias pelo teste de Tukey a 5\% de probabilidade (SAS INSTITUTE, 1996).

\section{RESULTADOS E DISCUSSÃO}

Verifica-se que a produção de matéria seca dos porta-enxertos de caramboleira apresentou diferença entre os tratamentos, sendo a solução nutritiva proposta por Hoagland \& Arnon (1950) inferior a de Furlani et al. (1999) e a de Castellane \& Araújo (1995), entretanto, não diferiu à solução de Sarruge (1975) (Tabela 2). Tais diferenças, na produção de matéria seca, podem ser atribuídas à associação das menores eficiências estudadas em cada solução utilizada.

Resultados divergentes foram obtidos por Franco \& Prado (2006) que, empregando as mesmas soluções, observaram semelhança no acúmulo de matéria seca total em mudas de goiabeira. De acordo com os autores, as concentrações e relações das soluções empregadas foram suficientemente adequadas para a exigência nutricional das plantas utilizadas.

TABELA 2 - Resumo da análise de variância (Q.M. e C.V.) para a variável matéria seca total dos portaenxertos de caramboleira, sob cultivo hidropônico aos 150 dias.

\begin{tabular}{lc}
\hline Soluções & Matéria seca total \\
\hline & $\mathrm{g}$ \\
Hoagland \& Arnon & $42,64^{\mathrm{b}}$ \\
Sarruge & $50,19^{\mathrm{ab}}$ \\
Castellane \& Araújo & $51,20^{\mathrm{a}}$ \\
Furlani & $53,04^{\mathrm{a}}$ \\
Q.M. & $62,75^{*}$ \\
C.V. $(\%)$ & 6,0 \\
\hline
\end{tabular}

* - Significativo a $5 \%$ de probabilidade. As médias seguidas da mesma letra, não diferem estatisticamente entre si, pelo teste Tukey a $5 \%$ de probabilidade. 
Observou-se que na presente pesquisa as plantas de caramboleira não apresentaram diferenças significativas à eficiência de absorção de $\mathrm{N}$ e de $\mathrm{Mg}$, quando submetidas às diferentes soluções nutritivas (Tabela 3 ).

Independentemente da solução, verificou-se que o nitrogênio e o potássio são os nutrientes mais requeridos na fase inicial de desenvolvimento da planta. É conhecida a atuação do nitrogênio na divisão celular e na produção de clorofila, bem como do potássio em processos osmóticos, na síntese de proteínas e na manutenção de sua estabilidade, na permeabilidade das membranas e no controle do pH (Epstein \& Bloom, 2006; Malavolta et al., 1997; Marschner, 1995). Justifica-se assim, a exigência da planta que se encontra em intensa atividade metabólica nesta fase inicial de desenvolvimento.

Considerando-se a eficiência de transporte como a capacidade da planta em transportar os nutrientes das raízes para a parte aérea, verificou-se que o Ca foi o nutriente que obteve os maiores índices, independentemente da solução nutritiva empregada (Tabela 4). Relato semelhante a este foi feito por Behling et al. (1989) que, empregando linhagens de tomateiro, atribuíram o fato às altas concentrações de $\mathrm{Ca}$ insolúvel nos tecidos da parte aérea da planta.

Para as soluções empregadas, com exceção do potássio que não apresentou diferença estatística significativa, aquelas propostas por Sarruge (1975) e Hoagland \& Arnon (1950) apresentaram significativa superioridade na eficiência de transporte de N, em relação às demais. Contudo, para o transporte de $\mathrm{P}, \mathrm{Ca}$ e $\mathrm{Mg}$ as soluções que se destacaram foram as de Hoagland \& Arnon (1950) e Furlani et al. (1999). Para o S, porém, a solução de Castellane \& Araújo (1995) diferiu-se estatisticamente superior à de Hoagland \& Arnon (1950) (Tabela 4).
Gratieri (2005), avaliando cultivares de pepino do grupo japonês, em diferentes soluções nutritivas, também não obteve uma única solução que melhor se correlacionasse com o número e a produtividade de frutos, bem como com o acúmulo de nutrientes na planta. Fernandes et al. (2002) avaliaram a produtividade, qualidade dos frutos e o estado nutricional de plantas de pepino, cultivadas em soluções nutritivas preparadas com diferentes fontes de fósforo, não detectando diferenças significativa entre os tratamentos. Furlani et al. (1999) relatam que trabalhos com soluções nutritivas empregando diversas formulações têm mostrado semelhanças, ou seja, não existe uma solução que se sobressaia à todas as outras. Epstein \& Bloom (2006) complementam que muitos esforços têm sido investidos na formulação de soluções nutritivas, mas há pouca probabilidade de que qualquer combinação particular de concentração e proporção de sais seja definitivamente superior para todas as situações e culturas, embora a pesquisa por tais melhoras seja uma busca constante ao longo do tempo.

Como no presente trabalho ofereceu-se o melhor ambiente possível às plantas, sem estresse fisiológico e, ou, falta de nutrientes, era de se esperar que não houvesse grandes diferenças entre as soluções na eficiência de utilização. Isso foi constatado pela não significância do N, $\mathrm{P}, \mathrm{K}$ e S frente aos tratamentos empregados. Entretanto, para a eficiência de utilização do cálcio, a solução de Hoagland \& Arnon (1950) foi inferior às demais (Tabela 5). Variações na eficiência de uso de cálcio em tecidos vegetais não são ainda bem compreendidas (Tomaz et al., 2003). A inativação do $\mathrm{Ca}$, devido à ligação e, ou, precipitação na forma de oxalato ou fosfato de cálcio, tem sido sugerida como causa para a baixa eficiência de utilização do nutriente (Behling et al., 1989; Horst et al., 1992).

TABELA 3 - Eficiência de absorção de nutrientes pelos porta-enxertos de caramboleira, sob cultivo hidropônico aos 150 dias.

\begin{tabular}{|c|c|c|c|c|c|c|}
\hline \multirow[t]{2}{*}{ Nutrientes } & \multicolumn{4}{|c|}{$\begin{array}{l}\text { Eficiência absorção } \\
\text { Soluções }\end{array}$} & \multirow[b]{2}{*}{ Q.M. } & \multirow[b]{2}{*}{ C.V. } \\
\hline & Hoagland & Sarruge & Castellane & Furlani & & \\
\hline \multicolumn{6}{|c|}{---------- mg nutriente / g de matéria seca raízes ----------- } & $(\%)$ \\
\hline $\mathrm{N}$ & 120,25 & 99,10 & 94,95 & 112,53 & $413,31^{\mathrm{ns}}$ & 11,0 \\
\hline $\mathrm{P}$ & $21,46 \mathrm{a}$ & $16,50 \mathrm{ab}$ & $15,18 \mathrm{~b}$ & $21,29 \mathrm{a}$ & $31,47^{*}$ & 12,3 \\
\hline K & $101,38 \mathrm{ab}$ & $86,13 b$ & $111,06 \mathrm{a}$ & $99,49 \mathrm{ab}$ & $316,15^{* *}$ & 6,1 \\
\hline $\mathrm{Ca}$ & $54,38 \mathrm{a}$ & $42,30 \mathrm{~b}$ & $39,69 b$ & $41,04 a$ & $137,53^{*}$ & 9,8 \\
\hline $\mathrm{Mg}$ & 20,15 & 18,98 & 15,89 & 20,91 & $16,64^{\mathrm{ns}}$ & 11,2 \\
\hline S & $13,92 \mathrm{ab}$ & $12,62 b$ & $13,35 b$ & $15,23 \mathrm{a}$ & $5,28^{* *}$ & 5,3 \\
\hline
\end{tabular}
mesma letra minúscula na horizontal, não diferem estatisticamente entre si, pelo teste Tukey a 5\% de probabilidade. 
TABELA 4 - Eficiência de transporte de porta-enxertos de caramboleira, sob cultivo hidropônico aos 150 dias.

\begin{tabular}{|c|c|c|c|c|c|c|}
\hline \multirow[t]{2}{*}{ Nutrientes } & \multicolumn{4}{|c|}{$\begin{array}{l}\text { Eficiência de transporte } \\
\text { Soluções }\end{array}$} & \multirow[b]{2}{*}{ Q.M. } & \multirow[b]{2}{*}{ C.V. } \\
\hline & Hoagland & Sarruge & Castellane & Furlani & & \\
\hline & ------ & 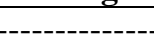 & & ----- & & $(\%)$ \\
\hline $\mathrm{N}$ & $439,24 \mathrm{ab}$ & $512,07 \mathrm{a}$ & $415,56 b$ & $406,23 b$ & $6888,40^{*}$ & 7,5 \\
\hline $\mathrm{P}$ & $241,96 a$ & $198,95 b$ & $200,69 b$ & $231,34 \mathrm{a}$ & $1414,35^{* *}$ & 5,3 \\
\hline $\mathrm{K}$ & 342,00 & 298,37 & 379,38 & 302,37 & $4333,17^{\mathrm{ns}}$ & 12,1 \\
\hline $\mathrm{Ca}$ & $973,64 a b$ & $927,71 b$ & $769,34 b$ & $1181,37 \mathrm{a}$ & $86934,28^{* *}$ & 9,8 \\
\hline $\mathrm{Mg}$ & $464,26 \mathrm{ab}$ & $442,77 b$ & $421,54 b$ & $521,21 \mathrm{a}$ & $5518,04^{* *}$ & 5,0 \\
\hline S & $280,73 b$ & $326,86 \mathrm{ab}$ & $353,24 a$ & $309,95 \mathrm{ab}$ & $2808,53^{*}$ & 7,3 \\
\hline
\end{tabular}

**; $* \mathrm{e}^{\mathrm{ns}}$ - Significativo a $1 \%$ e a $5 \%$ de probabilidade e não-significativo, respectivamente. As médias seguidas da mesma letra minúscula na horizontal, não diferem estatisticamente entre si, pelo teste Tukey a $5 \%$ de probabilidade.

TABELA 5 - Eficiência de utilização de porta-enxertos de caramboleira, sob cultivo hidropônico aos 150 dias.

\begin{tabular}{|c|c|c|c|c|c|c|}
\hline \multirow[t]{2}{*}{ Nutrientes } & \multicolumn{4}{|c|}{$\begin{array}{l}\text { Eficiência de utilização } \\
\text { Soluções }\end{array}$} & \multirow[t]{2}{*}{ Q.M. } & \multirow{2}{*}{$\frac{\text { C.V. }}{(\%)}$} \\
\hline & Hoagland & Sarruge & Castellane & Furlani & & \\
\hline $\mathrm{N}$ & $0,37 \mathrm{a}$ & $1,46 a$ & $0,50 \mathrm{a}$ & $0,46 a$ & $0,01^{\mathrm{ns}}$ & 11,7 \\
\hline $\mathrm{P}$ & $2,40 \mathrm{a}$ & $2,78 \mathrm{a}$ & $3,16 \mathrm{a}$ & $2,46 a$ & $0,37^{\mathrm{ns}}$ & 11,4 \\
\hline $\mathrm{K}$ & $0,44 \mathrm{a}$ & $0,53 a$ & $0,43 \mathrm{a}$ & $0,42 \mathrm{a}$ & $0,01^{\mathrm{ns}}$ & 10,2 \\
\hline $\mathrm{Ca}$ & $0,81 b$ & $1,07 \mathrm{a}$ & $1,21 \mathrm{a}$ & $1,26 \mathrm{a}$ & $0,12^{* *}$ & 7,3 \\
\hline $\mathrm{Mg}$ & $2,21 b$ & $2,39 b$ & $3,02 \mathrm{a}$ & $2,48 b$ & $0,36^{* *}$ & 6,7 \\
\hline $\mathrm{S}$ & $3,18 \mathrm{a}$ & $3,60 \mathrm{a}$ & $3,88 \mathrm{a}$ & $3,40 \mathrm{a}$ & $0,26^{\mathrm{ns}}$ & 8,7 \\
\hline
\end{tabular}

$* * ; * \mathrm{e}^{\mathrm{ns}}$ - Significativo a $1 \%$ e a $5 \%$ de probabilidade e não-significativo, respectivamente. As médias seguidas da mesma letra minúscula na horizontal, não diferem estatisticamente entre si, pelo teste Tukey a 5\% de probabilidade.

No caso do Mg, a solução de Castellane \& Araújo (1995) mostrou-se superior às demais. Santos (2000), em cultivo hidropônico de alface utilizando seis diferentes soluções nutritivas, concluiu que aquela proposta por Castellane \& Araújo (1995) apresentou produção superior às demais, bem como melhor aspecto qualitativo das plantas.

\section{CONCLUSÕES}

As soluções nutritivas de Furlani, de Castellane \& Araújo e de Sarruge foram semelhantes na produção de matéria seca de porta-enxertos de caramboleira. Sendo

O uso da solução nutritiva de Hoagland \& Arnon resultou em menor eficiência de utilização de $\mathrm{Ca}$ e $\mathrm{Mg}$ pelos porta-enxertos.

A eficiência de transporte dos nutrientes foi a mais afetada pelas soluções nutritivas utilizadas, enquanto a eficiência de utilização dos nutrientes sofreu pouca variação em função das diferentes soluções.

\section{REFERÊNCIAS BIBLIOGRÁFICAS}

ADAMS, P. Nutrition of greenhouse vegetables in NFT an hydroponic systems. Acta Horticulturae, n. 361, p. 254257, 1994.

BATAGLIA, O.C.; FURLANI, A.M.C.; TEIXEIRA, J.P.F.; FURLANI, P.R.; GALLO, J.R. Métodos de análise química de plantas. Campinas: Instituto Agronômico, 48p. (Boletim Técnico, 78), 1983.

BEHLING, J.P.; GABELMAN, W.H. \& GERLOFF, G.C. The distribution and utilization of calcium by two tomato (Lycopersicon esculentum Mill.) lines differing in calcium efficiency when grown under low-Ca stress. Plant Soil, v.113, p.189-196, 1989.

CASTELLANE, P.D.; ARAÚJO, J.A.C. de. Cultivo sem solo: hidroponia. 4.ed. Jaboticabal: FUNEP, 43p, 1995. 
DONADIO, L.C.; SILVA, J.A.A.; ARAÚJO, P.S.R.; PRADO, R.M. Caramboleira (Averrhoa carambola L.). Jaboticabal: Sociedade Brasileira de Fruticultura, v.1. 81p. 2001.

EPSTEIN, E. \& BLOOM, A.J. Nutrição mineral de plantas, princípios e perspectivas. Trad. Maria Edna Tenório Nunes -Londrina: Editora Planta, 86p, 2006.

FAGERIA, N.K. Otimização da eficiência nutricional na produção das culturas. Revista Brasileira de Engenharia Agrícola e Ambiental, v.2, n.1, p.6-16, 1998.

FERNANDES, A.A.; MARTIZEZ,H.E.P.; OLIVEIRA, L.R. Produtividade, qualidade dos frutos e estado nutricional de plantas de pepino, cultivados em hidroponia, em função das fontes de nutrientes. Horticultura Brasileira, Brasília, v.20, n.4, p.571-578, 2002.

FÖHSE, D.; CLAASEEN, N. \& JUNGK, A. Phosphorus efficiency of plants. Plant Soil, v.110, p.101-109, 1988.

FRANCO, C.F.; PRADO, R.M. Uso de soluções nutritivas no desenvolvimento e no estado nutricional de mudas de goiabeira: macronutrientes. Acta Scientiarum, Maringá, v. 28, n. 2, p. 199-205, 2006.

FURLANI, P.R.; SILVEIRA, L.C.P.; BOLONHEZI, D.; FAQUIN, V. Cultivo hidropônico de plantas. Campinas: IAC, 52 p. (Boletim Técnico 180), 1999.

GERLOFF, G.C. \& GABELMAN, W.H. Genetic basis of inorganic plant nutrition. In: Laüchli, A.; Bieleski, R.L. (Eds.). Inorganic plant nutrition. Encyclopedia of Plant Physiology. Berlim, New York, Tokyo: Springer-Verlag, v.15B, p.453-486, 1983 .

GRAHAM, R.D. Breeding for nutrition characteristics in cereals. In: TINKER, P.B.; LAUCHLI, A.(Eds.). Advances in plant nutrition. New York: praeger,p. 57-102, 1984.

GRATIERI, L.A. Avaliação de cultivares de pepino do grupo japonês, cultivados na fibra da casca de coco, sob diferentes soluções nutritivas. 2005, 58p. Dissertação (Mestrado em Produção Vegetal) - Faculdade de Ciências Agrárias e Veterinárias - UNESP, Jaboticabal, 2005.
HOAGLAND, D.R.; ARNON, D. I. The water culture method for growing plants without soils. Berkeley: California Agricultural Experimental Station, 347p., 1950.

HORST, W.J.; CURRLE, C. \& WISSEMEIER, A.H. Differences in calcium efficiency between cowpea (Vigna unguiculata (L.) Walp.) cultivars. Plant Soil, v.146, p.45-54, 1992.

LAUCHLI, A. Soil science in the next twenty five years: does a biotechnology play a role? Soil Science Society of American Journal, Madison, v.51,p.1405-1409,1987.

LI, B.; McKEAND, S.E.; ALLEN, H.L. Genetic variation in nitrogen use efficiency of loblolly pine seedlings. Forest Science, v.37, n.2, p.613-626, 1991.

LUCENA, J.J. Methods of diagnosis of mineral nutrition of plants: a critical review. Acta Horticulturae, v.448, p.179$182,1997$.

MALAVOLTA, E.; VITTI, G.C.; OLIVEIRA, S. Avaliação do estado nutricional das plantas. 2 ed. Piracicaba: POTAFÓS, 319p. 1997.

MARSCHNER, H. Mineral nutrition of higher plants. Academic Press, London, 889p. 1995.

SANTOS, O.S. Conceito, histórico e vantagens da hidroponia. In: SANTOS, O. Hidroponia da alface. Santa Maria: UFSM, p.5-9, 2000.

SARRUGE, J.R. Soluções nutritivas. Summa Phytopathologica, Jaboticabal, v.1, n.3, p.231-233, 1975.

SAS INSTITUTE. The SAS-system for windows: release 6.11 (software). Cary: Statistical Analysis System Institute, 1996.

SIDDIQI, M.Y.; GLASS, A.D.M. Utilization index: a modified approach to the estimation and comparison of nutrient efficiency in plants. Journal of Plant Nutrition, v.4, n.3, p.289-302, 1981.

SWIADER, J.M.; CHYAN, Y.; FREIJI, F.G. Genotypic differences in nitrate uptake and utilization efficiency in pumpkin hybrids. Journal of Plant Nutrition, v.17, n.10, p. 1687 - 1699, 1994. 
TÁVORA, F.J.A.F., FERREIRA, R.G.; HERNANDEZ, F.F.F.. Crescimento e relações hídricas em plantas de goiabeira submetidas a estresse salino com $\mathrm{NaCl}$. Revista Brasileira de Fruticultura, v.23, n.2, p.441- 446, 2001.
TOMAZ, M. A.; SILVA, S. R.; SAKIYAMA,N.S.; MARTINEZ, H. E. P. Eficiência de absorção, translocação e uso de cálcio, magnésio e enxofre por mudas enxertadas de Coffea arábica. Revista Brasileira Ciência Solo, Viçosa, v.27 n.5, p.885-892. 2003. 